\title{
A CMOS Inverter-Based Self-biased Fully Differential Amplifier
}

\author{
José Rui Custódio, Michael Figueiredo, Edinei Santin, and João Goes
}

\author{
Department of Electrical Engineering, Faculty of Sciences and Technology, Universidade Nova \\ de Lisboa, 2825-114 Monte de Caparica, Portugal \\ jrui.custodio@gmail.com, mf@uninova.pt, e.santin@fct.unl.pt, \\ jg@uninova.pt
}

\begin{abstract}
A CMOS self-biased fully differential amplifier is presented. Due to the self-biasing structure of the amplifier and its associated negative feedback, the amplifier is compensated to achieve low sensitivity to process, supply voltage and temperature (PVT) variations. The output common-mode voltage of the amplifier is adjusted through the same biasing voltages provided by the common-mode feedback (CMFB) circuit. The amplifier core is based on a simple structure that uses two CMOS inverters to amplify the input differential signal. Despite its simple structure, the proposed amplifier is attractive to a wide range of applications, specially those requiring low power and small silicon area. As two examples, a sample-and-hold circuit and a second order multi-bit sigmadelta modulator either employing the proposed amplifier are presented. Besides these application examples, a set of amplifier performance parameters is given.
\end{abstract}

Keywords: Fully-differential amplifier, inverter-based amplification, low power, self-biasing.

\section{Introduction}

The complementary fully differential amplifier has a wide range of applications in both the analog and digital domain. This type of amplifier may be used in applications where a wide input signal range is necessary, such as filters, voltage comparators, low voltage differential-signalling (LVDS) systems, and TTL-to-CMOS buffers. Examples of single-ended versions of this type of amplifier have been proposed in [1]. Besides a fully complementary structure, the amplifiers in [1] use a self-biasing method which avoids using separate biasing circuits to bias the amplifier. The advantages of self-biasing techniques are well-known: they simplify the implementation of amplifiers by removing amplifier biasing circuitry, thus saving power and die area; they enable circuits to be insensitive to process, supply voltage, temperature (PVT) and parameter variations; circuits employing these techniques are capable of supplying switching currents greater than the quiescent bias currents [1].

Similar to the amplifiers in [1], a single-ended self-biased amplifier was proposed in [2]. As in all single-ended amplifiers, they suffer from the lack of common-mode (CM) rejection and are highly sensitive to surrounding noisy circuitry. Although die area is practically doubled, the benefits of fully differential circuits outrun their disadvantages, thus it is always a better option than their single-ended counterpart. 
Fully differential self-biased amplifiers have already been proposed, such as, a folded cascode CMOS opamp (with and without gain boosting) [3], a class AB amplifier [4], and an LVDS signal receiver [5]. The amplifier reported in [4] is a pseudodifferential amplifier that relies on a complex active common-mode feedback (CMFB) network which has a separate biasing circuit, thus, is not completely selfbiased. The LVDS signal receiver from [5] uses a continuous time CMFB circuit with resistors, thus lowering the achievable gain.

The objective of this brief is to propose a simple CMOS inverter-based self-biased fully differential amplifier with constant DC gain over PVT variations for a wide range of applications. Basically this circuit is a differential version of the amplifier proposed in [1], where self-biasing and output common-mode adjustment is realized through a switched-capacitor (SC) CMFB network.

This paper is organized as follows. Section 2 debates on the contribution of technologic innovation introduced by the proposed circuit. The theory of operation and design analysis of the amplifier is described in Section 3. This section also proposes a design methodology. Application examples in a sample-and-hold amplifier and a sigma-delta $(\Sigma \Delta)$ modulator with simulation results are shown in Section 4 . The paper is completed with a section dedicated to the conclusions.

\section{Contribution to Technological Innovation}

It is well-known that circuits employing self-biasing are less sensitive to PVT variations [1]. In fact, these circuits have the advantage of being able to reestablish their nominal operating point after a perturbation in the operation. As a result, the yield of the designed circuits is improved substantially. Also, self-biased circuits do not necessitate dedicated biasing circuitry, and thus power and area are lowered.

Another desired characteristic of a circuit is the capability of being implemented differentially. Fully differential circuits suppress even-order harmonic distortion and are more robust to extrinsic (surrounding) noise. This is a major concern in mixed-signal circuits, where the noise from digital circuitry is coupled into the sensitive analog blocks via the substrate and supply lines. Implementation simplicity (in terms of structure, number of devices, and layout effort) is another issue, since, generally, it leads to circuits consuming less power and area, presenting low noise, and easy to be laid out.

In this work we present a CMOS inverter-based self-biased fully-differential amplifier with the innovation of using a simple SC CMFB circuit to both control the output $\mathrm{CM}$ voltage and bias the amplifier. The proposed amplifier accomplishes the above mentioned characteristics and is well suited for a broad range of applications as will be demonstrated.

\section{Inverter-Based Self-biased Fully Differential Amplifier}

\subsection{Theory of Operation}

The proposed amplifier, illustrated in Fig. 1, comprises two input CMOS inverters $\left(M_{2}, M_{3}\right)$ and two voltage controlled resistors (VCR) $M_{1}$ and $M_{4}$, biased in the boundary of the saturation and triode regions (it is very difficult to bias both these 
transistors simultaneously in the saturation region). A simple SC CMFB circuit comprising only two capacitors $\left(C_{C M}\right)$ and five switches is used to properly adjust the output common-mode voltage to about $V_{D D} / 2$ and, on the other hand, to provide closed-loop control (self-biasing) of the amplifier. The VCRs, $M_{1}$ and $M_{4}$, have their gate voltages controlled by the output of the SC CMFB circuit, $V_{C M} . M_{1}$ and $M_{4}$ together with $V_{C M}$ are connected to the input inverters in a negative feedback loop reducing the effect of PVT variations on the amplifier's DC gain. As an example of compensation, if $V_{D D}$ increases, the source-gate voltage in PMOS device $M_{1}, V_{S G P 1}$, also increases producing an increase in the bias current $I_{D}$. This increase will change proportionally the current in the two input inverters, increasing the output CM voltage. As a consequence, the CMFB circuit will produce a higher $V_{C M}$ output control voltage (assuming that $V_{C M I}$ is constant and provided by a bandgap circuit) forcing $V_{G S P 1}$ to remain constant, thus compensating the $V_{D D}$ variation. Process and temperature variations have similar compensations through the negative-feedback loop. As will be seen in the following sub-section, the DC gain, $A_{V 0}$, is approximately the ratio of a transconductance by an output conductance, $\mathrm{gm} / \mathrm{gds}$. The DC gain is maintained constant because variations in $g m( \pm 30 \%)$ are accompanied by similar variations in $g d s$, thus their ratio has variations less than $7 \%$ over PVT variations.

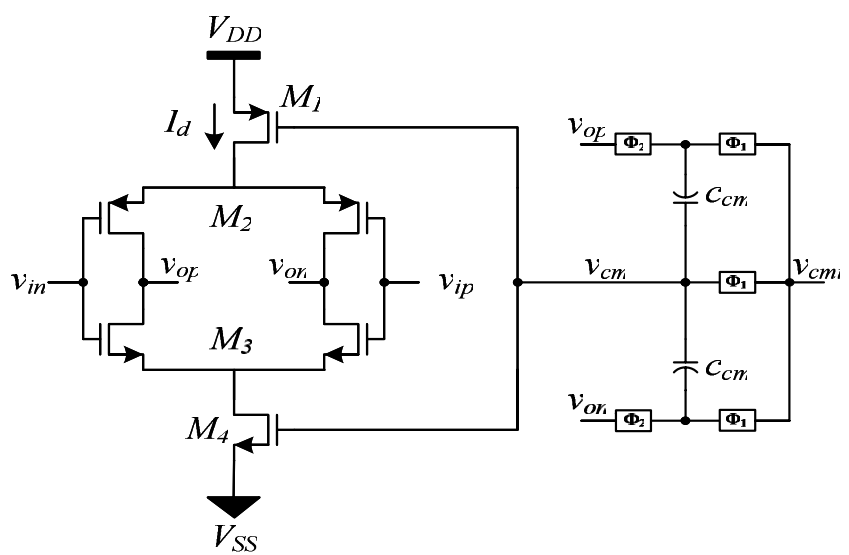

Fig. 1. Schematic of the fully-differential single-stage self-biased amplifier with the SC CMFB circuit

\subsection{Design Analysis}

Performing a small signal analysis followed by a Y-parameter simplification of the amplifier it is possible to obtain the amplifier's transfer function, given by,

$$
A_{V} \cong \frac{s \cdot\left(c g d_{2}+c g d_{3}\right)-g m_{3}-g m_{2}}{s \cdot\left(c g d_{2}+c g d_{3}+C_{L}+C_{C M}\right)+g d s_{2}+g d s_{3}}
$$

Equation (2) presents the amplifier's DC gain. Some assumptions have been made, namely, $g m_{3} \cong k \cdot g m_{2}=k \cdot g m\left(k=\mu_{N} / \mu_{P}\right), b_{e f 2} \cong b_{e f 3}=b_{e f}\left(b_{e f} \cong 0.85\right)$ and $g d s_{2} \cong g d s_{3}=$ $g d s . \mu_{N}$ and $\mu_{P}$ represent the mobilities of the NMOS and PMOS transistors, 
respectively. $b_{e f}$ represents the $g m$ degradation in devices $M_{2}$ and $M_{3}$ due to bodyeffect. Special care should be taken when considering a value for $b_{e f}$, as it may vary with the operating region of each transistor.

$$
A_{V 0} \cong \frac{b_{e f} \cdot g m \cdot(1+k)}{2 \cdot g d s}
$$

It should be noticed that (1) represents the gain of a standard inverter input amplifier, in other words, no parameters of devices $M_{1}$ and $M_{4}$ appear in the transfer function.

From (1) it is also possible to obtain expressions for the dominant pole frequency (BW), gain-bandwidth product (GBW), and the unity gain frequency (UGF), which are important performance parameters in opamp circuits. The following equations respectively represent the mentioned performance parameters. Parasitic capacitor $c g d$ $=c g d_{2}=c g d_{3}$ and $C_{L}$ represents the output load capacitance.

$$
\begin{gathered}
f_{\text {pole }}=B W=\frac{2 \cdot g d s}{C_{L}+C_{C M}+2 \cdot c g d} \\
G B W=A_{V 0} \times B W=\frac{b_{e f} \cdot g m \cdot(1+k)}{C_{L}+C_{C M}+2 \cdot \operatorname{cgd}} \\
U G F=\frac{b_{e f} \cdot g m \cdot(1+k)-2 \cdot g d s}{C_{L}+C_{C M}}
\end{gathered}
$$

By considering the DC gain and GBW as initial specifications for the opamp, a possible design procedure would be to use (4) to obtain the $g m$ that satisfies the GBW for a given effective load $\left(C_{e f f}=C_{L}+C_{C M}+2 c g d\right)$, and then to employ (2) to find the $g d s$ that satisfies the $A_{V 0}$ requirement. The drain currents for $M_{2}$ and $M_{3}$ can be calculated using $g m$ and a given drain-source saturation voltage, $V_{D S s a t}=V_{G S}-V_{T} \geq 100 \mathrm{mV}$. Finally, $M_{2}$ and $M_{3}$ should be sized to be in saturation and to have an adequate $g d s$ (adjusted by the channel lengths). Multiplying the drain current of $M_{2}$ (or $M_{3}$ ) by two (to account for both current paths, differential circuit) it is possible to size $M_{1}$ and $M_{4}$ in the triode/saturation boundary region for a $V_{D S s a t} \geq 100 \mathrm{mV}\left(V_{D S s a t 1,4}\right.$ should not be large to avoid degrading output swing). Some care should be taken in the sizing of the transistors to guarantee the desired output common-mode voltage, power budget, output swing and PVT and mismatch insensitivity.

\section{Simulation Results}

This section presents simulation results of the proposed amplifier for two target applications. Firstly, the amplifier is applied in a sample-and-hold amplifier (SHA) intended for the front-end stage of a low resolution, high speed pipelined analog-to-digital converter (ADC). Secondly, the proposed amplifier is used in a multi-bit $\Sigma \Delta$ modulator targeted at hearing aid devices. Both amplifiers were designed in a $130 \mathrm{~nm}$ 
high speed $1.2 \mathrm{~V}$ CMOS technology $\left(L_{\min }=120 \mathrm{~nm}\right)$. The mobility and threshold parameters (Level 2), $K_{N}, K_{P}, V_{T N}$ and $V_{T P}$ of the devices are, respectively, $525 \mu \mathrm{AV}^{-2}$, $145 \mu \mathrm{AV}^{-2}, 0.38 \mathrm{~V}$ and $-0.33 \mathrm{~V}$. For $V_{c m i}, 550 \mathrm{mV}$ was used.

\subsection{Application in a Sample-and-Hold Amplifier}

An interesting application for the proposed amplifier is in a SHA as depicted in Fig. 2 . This SHA was successfully applied in the front-end stage of a low resolution (7 bit) and high speed $(500 \mathrm{MS} / \mathrm{s})$ pipelined ADC. As a result, the basic specifications of the amplifier, such as DC gain $\left(A_{V 0}\right)$ and GBW, should be large enough to accommodate these requirements. Furthermore, the power consumption must be kept as low as possible to avoid degradation in the ADC performance.

With these prerequisites in mind and by using the proposed design procedure as an initial step followed by electrical simulations for sizing refinements (this includes typical and, at least, process corners), the transistors are sized as reported in Table 1.

Table 1. Feature size of the transistors for the sample-and-hold amplifier

\begin{tabular}{ll}
\hline Transistor & $W(\mu \mathrm{m}) / L(\mu \mathrm{m})$ \\
\hline$M_{1}$ & $47 / 0.18$ \\
$M_{2}$ & $32 / 0.16$ \\
$M_{3}$ & $32 / 0.16$ \\
$M_{4}$ & $32 / 0.50$ \\
\hline
\end{tabular}

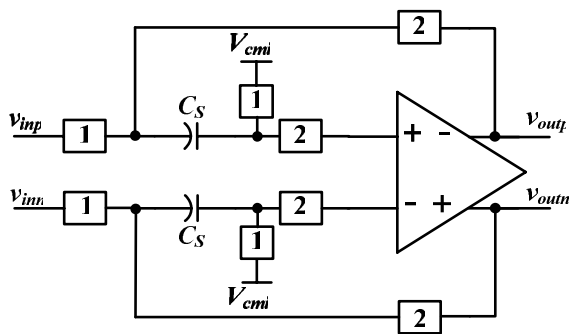

Fig. 2. Complete electrical schematic of the sample-and-hold amplifier

To evaluate the overall performance and robustness of the designed amplifier, its key parameters are simulated for different PVT corners according to Table 2. At typical conditions $(\mathrm{Ct})$, the amplifier dissipates $0.9 \mathrm{~mW}$ and achieves a DC gain of 25.8 $\mathrm{dB}$ with a GBW larger than $2.88 \mathrm{GHz}$ (for an effective load capacitance of $0.5 \mathrm{fF}$ ). The results for the other PVT corners are shown in Fig. 3. It should be noted that, although the GBW and the static power vary considerably, the DC gain varies less than $\pm 0.75 \mathrm{~dB}$ considering all corners. These results were expected and clearly indicate the contribution of self-biasing in reducing amplifier sensitivity to PVT variations, concerning the DC gain. 
Table 2. List of PVT corners used in the electrical simulations

\begin{tabular}{llll}
\hline Corner & Process & Supply Voltage & Temperature \\
\hline $\mathrm{Ct}$ & TT & 1.2 & 27 \\
$\mathrm{C} 1$ & TT & 1.14 & 27 \\
$\mathrm{C} 2$ & TT & 1.26 & 27 \\
$\mathrm{C} 3$ & SS & 1.14 & -40 \\
$\mathrm{C} 4$ & SS & 1.14 & 85 \\
$\mathrm{C} 5$ & SS & 1.26 & -40 \\
$\mathrm{C} 6$ & SS & 1.26 & 85 \\
$\mathrm{C} 7$ & FF & 1.14 & -40 \\
C8 & FF & 1.14 & 85 \\
C9 & FF & 1.26 & -40 \\
C10 & FF & 1.26 & 85 \\
\hline
\end{tabular}
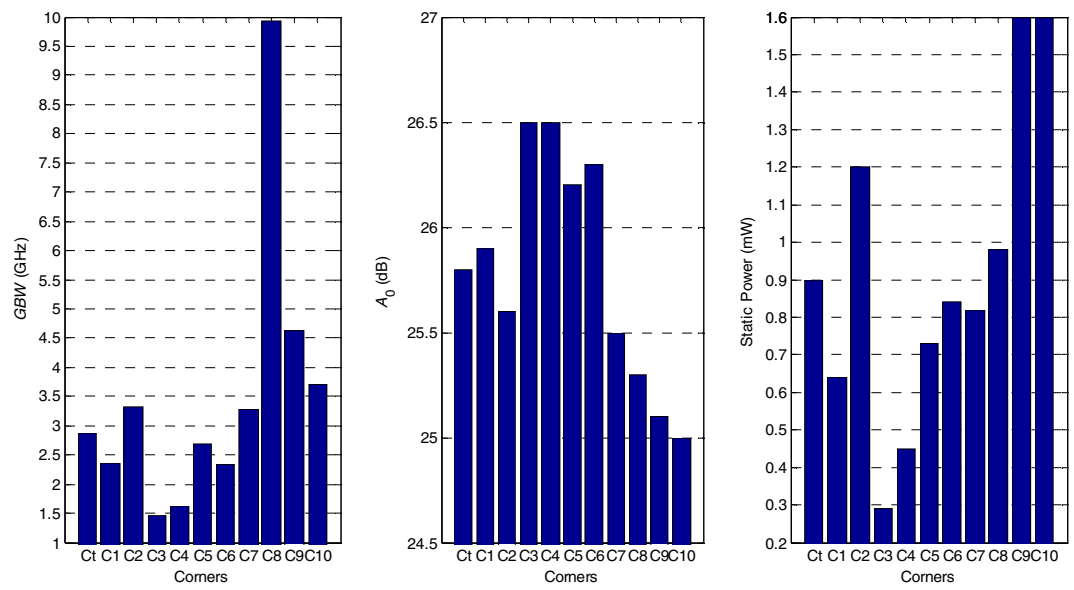

Fig. 3. GBW, $\mathrm{A}_{0}$, and static power of the amplifier for PVT corners listed in Table 2

\subsection{Application in a Multi-bit Sigma-Delta Modulator}

The proposed amplifier was also applied to a second-order four-bit $\Sigma \Delta$ modulator as shown in Fig. 4. In this context, the amplifier is employed to realize a low power SC integrator, which is one of the key elements that determine the global performance of the $\Sigma \Delta$ modulator. This modulator is intended for hearing aids, and, therefore, it requires a dynamic range of about $96 \mathrm{~dB}$ with a peak signal-to-(noise plus distortion) ratio (SNDR) above $60 \mathrm{~dB}$. Additionally, the signal bandwidth is $20 \mathrm{kHz}$. These specifications are met using a second-order four-bit $\Sigma \Delta$ modulator with nonlinear digital-to-analog converters with an oversampling ratio of 32 .

The most stringent requirements are needed in the first integrator, which results in a higher complex amplifier design than necessary for the second integrator. It was verified by high level simulations that a minimum DC gain of $40 \mathrm{~dB}$ in the amplifier of the first integrator is enough to achieve the target dynamic range of $96 \mathrm{~dB}$. In addition, the amplifier needs to settle its output with an error lower than 10 bit of resolution required 
for $60 \mathrm{~dB}$ of SNDR when operated at $1.28 \mathrm{MHz}$ sampling frequency. This is achieved with a GBW higher than $8.4 \mathrm{MHz}$. To meet all these specifications the amplifier is sized following the same procedure described earlier and the results are shown in Table 3.

Table 3. Feature size of the transistors for the amplifier of the first integrator

\begin{tabular}{ll}
\hline Transistor & $W(\mu \mathrm{m}) / L(\mu \mathrm{m})$ \\
\hline$M_{1}$ & $8.5 / 1.2$ \\
$M_{2}$ & $15 / 0.8$ \\
$M_{3}$ & $16 / 1.3$ \\
$M_{4}$ & $7.75 / 4.5$ \\
\hline
\end{tabular}

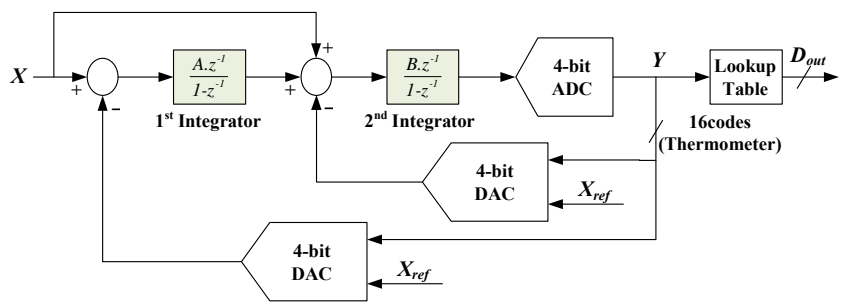

Fig. 4. Complete block diagram of the second-order four-bit $\Sigma \Delta$ modulator

The performance of the amplifier was verified through different PVT corners simulations as indicated in Table 2. The amplifier achieves a DC gain of $42.9 \mathrm{~dB}$ with a GBW larger than $8.4 \mathrm{MHz}$ (for an effective load capacitance of $10 \mathrm{pF}$ ) dissipating only $41 \mu \mathrm{W}$ at typical operation. The results for the remaining corners are depicted in Fig. 5. As in the previous example, we can note that the DC gain varies less than \pm 1.5 $\mathrm{dB}$ considering all corners.
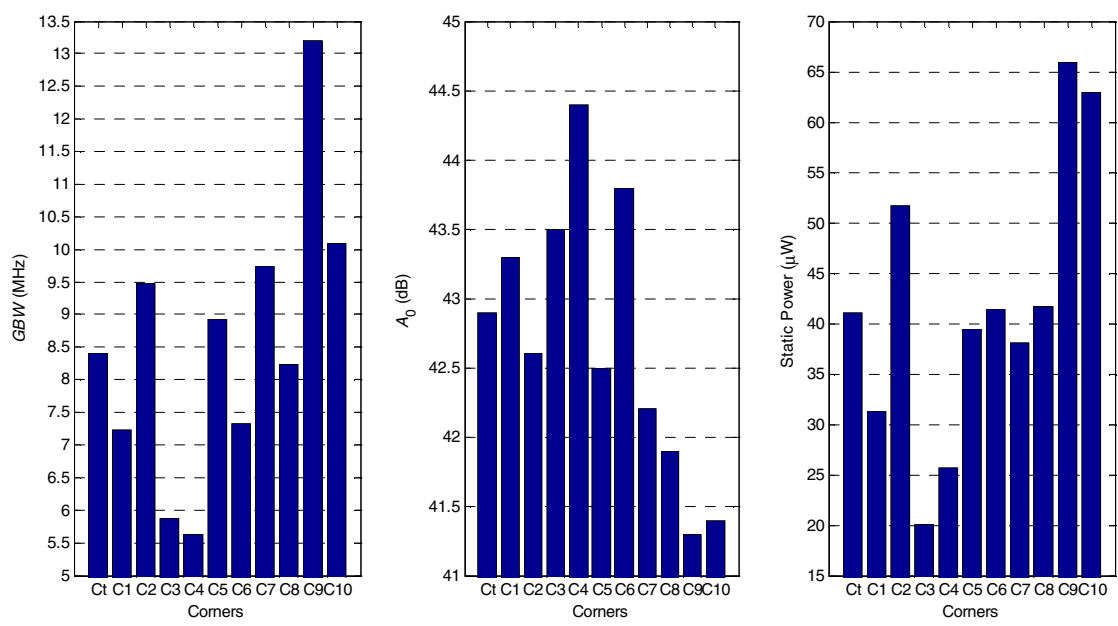

Fig. 5. GBW, $\mathrm{A}_{0}$, and static power of the amplifier for PVT corners listed in Table 2 


\section{Conclusions}

A CMOS self-biased fully differential amplifier was presented. In order to generate the biasing voltages of the amplifier, a CMFB is employed. The CMFB circuit, based on an SC network, uses the amplifier's outputs to derive the mentioned voltages, hence the designation, self-biased amplifier. Due to the self-biasing structure of the amplifier and its associated negative feedback, the amplifier is compensated to achieve low sensitivity to PVT variations. The output common-mode voltage of the amplifier is adjusted through the same biasing voltages provided by the CMFB circuit. The amplifier core is based on a simple structure that uses two CMOS inverters to amplify the input differential signal. Despite its simple structure, the proposed amplifier is attractive to a wide range of applications, specially those requiring low power and small area. As two examples, a second order $\Sigma \Delta$ modulator and a SHA circuit either employing the proposed amplifier were presented. Besides these application examples, a set of amplifier performance parameters was given.

Acknowledgments. This work was supported in part by the Portuguese Foundation for Science and Technology (FCT/MCTES) under projects SPEED (PTDC/EEAELC/ 66857/2006), LEADER (PTDC/EEA-ELC/69791/2006) and IMPACT (PTDC/EEAELC/101421/2008). The authors also would like to thank Marta Kordasz for implementing an opamp transfer function extractor program based on Y-parameters, which greatly reduced circuit analysis time.

\section{References}

1. Bazes, M.: Two Novel Fully Complementary Self-Biased CMOS Differential Amplifiers. J. of Solid-State Circuits 26, 165-168 (1991)

2. Zhu, Z., Liu, Y., Yang, Y.: A High Speed Self-biased CMOS Amplifier IP core. In: Proc. IEEE International Workshop on VLSI Design and Video Technology, pp. 6-9 (2005)

3. Mandal, P., Visvanathan, V.: A Self-Biased High Performance Folded Cascode CMOS Op-Amp. In: Proc. 10th International Conference on VLSI Design, pp. 429-434 (1997)

4. Giustolisi, G., Grasso, A.D., Pennisi, S.: High-Drive and Linear CMOS Class-AB PseudoDifferential Amplifier. IEEE Trans. on Circuits and Systems II 54(2), 112-116 (2007)

5. Xiao, P., Kuchta, D., Stawiasz, K., Ainspan, H., Choi, J.-H., Shin, H.: A 500 Mb/s, 20Channel CMOS Laser Diode Array Driver for a Parallel Optical Bus. IEEE ISSCC Digest of Technical Papers 467, 250-251 (1997) 